\title{
High mitochondrial calcium levels precede neuronal death in vivo in Alzheimer's disease
}

\author{
Maria Calvo-Rodriguez ${ }^{1}$ and Brian J. Bacskai ${ }^{1, *}$ \\ ${ }^{1}$ Alzheimer Research Unit, Department of Neurology, Massachusetts General Hospital and Harvard Medical School, 114, 16th St, \\ Charlestown, MA 02129, USA. \\ * Corresponding Author: \\ Brian J. Bacskai, Alzheimer Research Unit, Department of Neurology, Massachusetts General Hospital and Harvard Medical School \\ 114, 16th St, Charlestown, MA 02129, USA; E-mail: BBACSKAI@mgh.harvard.edu
}

\begin{abstract}
Alzheimer's disease (AD), the most common cause of dementia, affects millions of people worldwide. Suggested mechanisms of neurotoxicity in AD include impaired calcium $\left(\mathrm{Ca}^{2+}\right)$ homeostasis and mitochondrial dysfunction, both contributing to neuronal damage. Little was known about the exact mitochondrial $\mathrm{Ca}^{2+}$ homeostasis in the living brain, particularly in AD. Only now, with the development of intravital imaging techniques and transgenic mouse models of the disease, we are able to directly observe $\mathrm{Ca}^{2+}$ levels in specific regions or particular subcellular compartments of cells, such as mitochondria. Using multiphoton microscopy, a $\mathrm{Ca}^{2+}$ reporter targeted to mitochondria and a mouse model of cerebral $\beta$ amyloidosis (APP/PS1), our recent study (Nat Comms 2020, 11:2146) found elevated mitochondrial $\mathrm{Ca}^{2+}$ concentration in the transgenic mouse after plaque deposition, and after topical application of natural soluble amyloid beta $(A \beta)$ oligomers to the healthy mouse brain at concentrations similar to those found in the human brain. Elevated $\mathrm{Ca}^{2+}$ in mitochondria preceded neuronal death and could be targeted for neuroprotective therapies in AD. Here, we describe our main findings and pose new questions for future studies aimed at better understanding mitochondrial $\mathrm{Ca}^{2+}$ dyshomeostasis in AD.
\end{abstract}

\section{MITOCHONDRIAL CALCIUM OVERLOAD AFTER PLAQUE DEPOSITION}

$\mathrm{Ca}^{2+}$ is essential for the normal function of mitochondrial activity, and therefore $\mathrm{Ca}^{2+}$ levels are tightly regulated in mitochondria. However, excessive levels of $\mathrm{Ca}^{2+}$ in mitochondria, i.e. mitochondrial $\mathrm{Ca}^{2+}$ overload, can lead to an increase of reactive oxidative stress (ROS) species, de- crease of ATP production and eventually to cell death via apoptosis. Cytosolic $\mathrm{Ca}^{2+}$ homeostasis is impaired in $\mathrm{AD}$, as evidenced by hyperactive neurons close to amyloid plaques or high cytosolic $\mathrm{Ca}^{2+}$ in transgenic mice with plaque deposition. But, is mitochondrial $\mathrm{Ca}^{2+}$ homeostasis also impaired in vivo in $\mathrm{AD}$ ? Are $\mathrm{Ca}^{2+}$ levels increased or diminished in the neurons? In vitro studies suggested that mitochondrial $\mathrm{Ca}^{2+}$ homeostasis was altered in the presence of $A \beta$ oligomers $(A \beta O)$, but these questions remained unresolved in vivo. To address this issue, we used a transgenic mouse model of cerebral amyloidosis, APP/PS1 - a model that develops amyloid plaques comparable to those from human patients starting at around four months of age - and intravital imaging with multiphoton microscopy. To measure $\mathrm{Ca}^{2+}$ concentration in mitochondria, we used a ratiometric $\mathrm{Ca}^{2+}$ reporter (Yellow Cameleon, YC) targeted to neuronal mitochondria (hSyn.2mt.YC3.6), that we injected as adenovirus (AAV) in the somatosensory cortex of the mice. This ratiometric reporter, based on Förster resonance energy transfer (FRET), allows for quantitative measurement of absolute $\mathrm{Ca}^{2+}$ concentration and direct comparison between cells and compartments. We observed mitochondrial $\mathrm{Ca}^{2+}$ overload in the neurons of old APP/PS1 transgenic mice, once plaque deposition had started, unlike the young transgenic mice (pre-pathology). Transgenic mice showed twice as many mitochondria with high $\mathrm{Ca}^{2+}$ compared to the wild-type (Wt) mice. This difference was found in both somas and neurites. Other studies have previously reported no changes in cytosolic $\mathrm{Ca}^{2+}$ or neuronal function before plaque deposition, supporting the notion that mitochondrial $\mathrm{Ca}^{2+}$ overload in neurons follows, rather than precedes, amyloid plaque deposition. Figure 1 shows an example of in vivo multiphoton microscopy images of mitochondria in neurons in APP/PS1 mouse and in Wt mouse after transduction of the AAV. We did not

MICROREVIEW on: Calvo-Rodriguez M, Hou SS, Snyder AC, Kharitonova EK, Russ AN, Das S, Fan Z, Muzikansky A, Garcia-Alloza M, SerranoPozo A, Hudry E, Bacskai BJ (2020). Increased mitochondrial calcium levels associated with neuronal death in a mouse model of Alzheimer's disease. Nat Commun 11(1):2146. doi: 10.1038/s41467-020-16074-2 


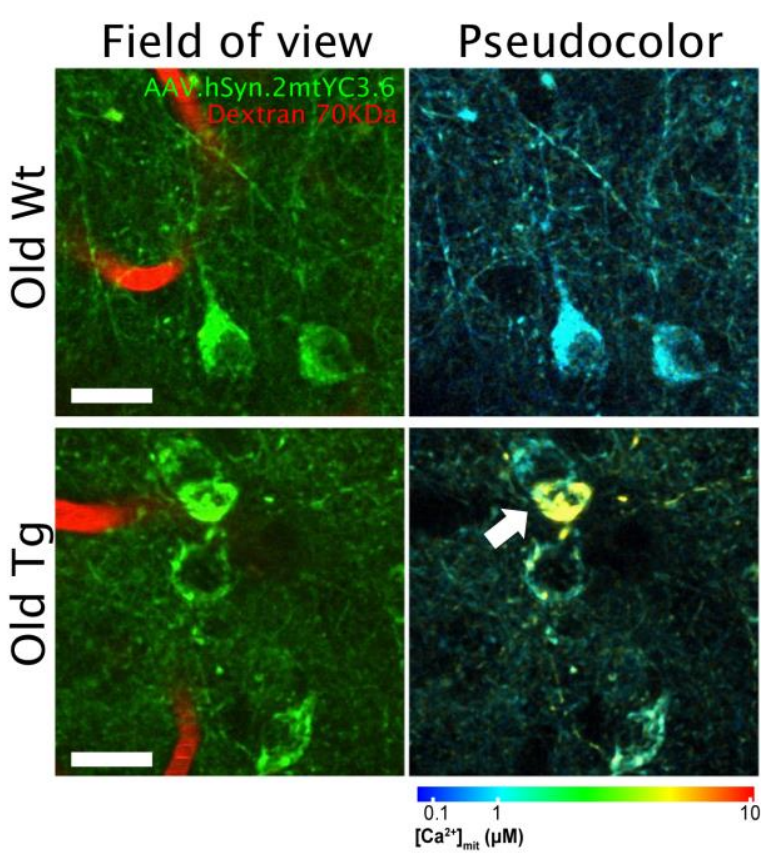

FIGURE 1: In vivo multiphoton microscopy images of mitochondrial $\mathrm{Ca}^{2+}$ in neurons in the Wt and APP/PS1 mice. Mice were injected with AAV.hSyn.2mtYC3.6 and a cranial window was implanted. Mitochondrial $\mathrm{Ca}^{2+}$ was later evaluated with multiphoton microscopy. Pictures are representative of old wild-type (Wt, top) and transgenic mice (APP/PS1 Tg, bottom). The field of view shows the AAV transduction in neuronal mitochondria (green) and the blood vessels labeled with fluorescent Dextran (red). Pseudocolor images represent the color coded mitochondrial $\mathrm{Ca}^{2+}$ concentrations for the corresponding field of view images. Arrow points to a neuron with mitochondrial $\mathrm{Ca}^{2+}$ overload. Scale bar represents $20 \mu \mathrm{m}$.

find any correlation between mitochondrial $\mathrm{Ca}^{2+}$ overload and the distance to plaques, suggesting that diffusible soluble $A \beta$ species ( $A \beta$ oligomers, $A \beta O$ ), rather than individual plaques, are responsible for the mitochondrial $\mathrm{Ca}^{2+}$ increase in the neurons of the transgenic mouse.

\section{SOLUBLE A $\beta$ OLIGOMERS INCREASE MITOCHONDRIAL CALCIUM IN NEURONS VIA THE MCU}

To confirm that soluble $A \beta$ species drive the mitochondrial $\mathrm{Ca}^{2+}$ increase in neurons, we topically applied naturally secreted $A \beta o$ (obtained from primary cortical neurons isolated from transgenic embryos, referred to as transgenic conditioned media, $\operatorname{TgCM}$ ) at concentrations similar to those found in the human AD brain directly onto the surface of the living brain of a Wt naive mouse (C57BL/6J) expressing the adenovirus hSyn.2mtYC3.6. Application of $\mathrm{TgCM}$ transiently increased mitochondrial $\mathrm{Ca}^{2+}$ concentration in the neurons, whereas Wt conditioned media (obtained from Wt littermates) or A $\beta$-immunodepleted TgCM did not. Neurites were more susceptible to this increase than somas. These results confirmed our previous observations in the APP/PS1 transgenic mouse, and implicated soluble $A \beta O$ in the mitochondrial $\mathrm{Ca}^{2+}$ dyshomeostasis in vivo. After returning to normal mitochondrial $\mathrm{Ca}^{2+}$ levels, we observed that mitochondrial shape and size were altered - mitochondria were smaller and rounded -, suggesting that the toxic effects of $A \beta o$ in vivo were not only restricted to mitochondrial $\mathrm{Ca}^{2+}$, but also to mitochondrial morphology. Additionally, this suggests that the change in size and shape is a secondary event after a mitochondrial $\mathrm{Ca}^{2+}$ increase. It is believed that mitochondrial fragmentation precedes neuronal cell death, which establishes a connection with mitochondrial $\mathrm{Ca}^{2+}$. Interestingly, the APP/PS1 transgenic mouse also exhibits fragmented and swollen mitochondria once the pathology is present, and the brains from $A D$ patients show impaired balance of mitochondrial fission and fusion proteins.

How does $A \beta$ induce mitochondrial $\mathrm{Ca}^{2+}$ uptake? And is it possible to inhibit it in order to prevent or decrease the mitochondrial $\mathrm{Ca}^{2+}$ overload? The mitochondrial $\mathrm{Ca}^{2+}$ uniporter (MCU) complex is the main pathway for mitochondrial $\mathrm{Ca}^{2+}$ influx. However, the mechanism by which $A \beta$ induces a $\mathrm{Ca}^{2+}$ increase in neuronal mitochondria in vivo was not previously known. Various hypotheses proposed that $A \beta$ could create pores in membranes, form a $\mathrm{Ca}^{2+}$ permeable pore itself or act on native channels. In this study, we demonstrated that blocking the MCU complex in vivo with Ru360 - a specific blocker of the channel - prevented the mitochondrial $\mathrm{Ca}^{2+}$ uptake elicited by $\mathrm{TgCM}$. To confirm that Ru360 application did not render neurons insensitive to $A \beta$ or blocked the $\mathrm{Ca}^{2+}$ influx channels of the plasma membrane, we measured the cytosolic $\mathrm{Ca}^{2+}$ influx induced by $\mathrm{A} \beta \mathrm{O}$ with the same $\mathrm{Ca}^{2+}$ reporter (YC) targeted to the cytosol of neurons (CBA.YC3.6). We observed that Ru360 did not interfere with the rise in the cytosolic $\mathrm{Ca}^{2+}$ induced by $A \beta$. These results suggest that $M C U$ is required for the increase in mitochondrial $\mathrm{Ca}^{2+}$ induced by $A \beta$ in vivo, and points to $\mathrm{MCU}$ as a potential target candidate for $A D$.

\section{HIGH MITOCHONDRIAL CALCIUM PRECEDES NEU- RONAL DEATH IN VIVO}

What are the implications for increased neuronal mitochondrial $\mathrm{Ca}^{2+}$ in the in vivo brain? We observed that in the APP/PS1 transgenic mouse, the small fraction of neurons with elevated mitochondrial $\mathrm{Ca}^{2+}$ in the soma died within 24 hours. Their nuclei became condensed (a sign of apoptosis) and the AAV fluorescence in the soma was lost. This effect did not happen in cells with normal mitochondrial $\mathrm{Ca}^{2+}$ levels. Rapid neuronal death was previously observed in the APP/PS1 model following increased oxidative stress in the cytosol. Our in vitro data showed that, in addition, soluble $A \beta$ triggered opening of the mitochondrial permeability transition pore (mPTP), a decrease of the mitochondrial membrane potential and activation of caspases, events that were secondary to the increase in mitochondrial $\mathrm{Ca}^{2+}$. All these findings together link high mitochondrial $\mathrm{Ca}^{2+}$, oxidative stress and neuronal cell death via apoptosis in vivo in AD. 


\section{MITOCHONDRIAL CALCIUM DYSREGULATION IN THE HUMAN ALZHEIMER'S BRAIN}

Lastly, is mitochondrial $\mathrm{Ca}^{2+}$ homeostasis impaired in the human Alzheimer's brain? Because direct analysis of mitochondrial $\mathrm{Ca}^{2+}$ levels in the human brain is not feasible, could we evaluate the proteins or channels involved in mitochondrial $\mathrm{Ca}^{2+}$ transport in the human brain? And if we can, to what extend are they affected? Using data publicly available at the AMP-AD (Accelerating Medicines Partnership-Alzheimer's disease) knowledge portal, we evaluated the differential expression of genes encoding proteins involved in the mitochondrial $\mathrm{Ca}^{2+}$ transport. $\mathrm{Ca}^{2+}$ influx into mitochondria is mainly led by the MCU complex, which includes the MCU pore, the regulatory subunits MICU1 and MICU2, the essential regulator EMRE, and other proteins such as MCUb and MICU3. $\mathrm{Ca}^{2+}$ efflux from mitochondria is primarily controlled by the $\mathrm{Na}^{+} / \mathrm{Ca}^{2+}$ exchanger (NCLX). We compared the expression of the genes encoding these proteins between $A D$ subjects and control individuals using microarray and RNA-seq datasets from the MSBB (Mount Sinai Brain Bank) and the ROSMAP (Religious Orders Study and Memory and Aging Project) cohorts. We observed that the MCU and all subunits of the complex $-\mathrm{Ca}^{2+}$ influx were downregulated in AD compared with control subjects, whereas $\mathrm{NCLX}-\mathrm{Ca}^{2+}$ efflux - was the only one upregulated.
These results suggest a dysregulation in the human genes encoding for mitochondrial $\mathrm{Ca}^{2+}$ transport, and imply a counteracting effect to avoid mitochondrial $\mathrm{Ca}^{2+}$ overload in the neurons in the AD human brain.

This study helped clarify the understanding of the dynamics of mitochondrial $\mathrm{Ca}^{2+}$ in $A D$. It suggests that $A \beta$ aggregates (particularly soluble $A \beta$ ) increase mitochondrial $\mathrm{Ca}^{2+}$ in $\mathrm{AD}$ via the $\mathrm{MCU}$ in vivo, an effect that is linked to neuronal cell death via apoptosis (Figure 2). We demonstrated that the extent of mitochondrial $\mathrm{Ca}^{2+}$ alterations are spatially restricted in the living brain and highlight the need for imaging studies that can probe these anatomically specific effects. However, this work highlights other questions that remain unresolved. Does mitochondrial $\mathrm{Ca}^{2+}$ elevation occur concomitantly with plaque formation? Is it possible to observe mitochondrial morphological abnormalities and altered dynamics (fission, fusion and transport) induced by $A \beta$ in vivo? Does tau have any effect on mitochondria, both in terms of $\mathrm{Ca}^{2+}$ abnormalities and motility? Additionally, our studies point to $\mathrm{MCU}$ as a possible therapeutic target for $A D$ and other neurodegenerative diseases in which mitochondrial $\mathrm{Ca}^{2+}$ is impaired, however, further efforts will be needed to unveil the clinical relevance of our findings.

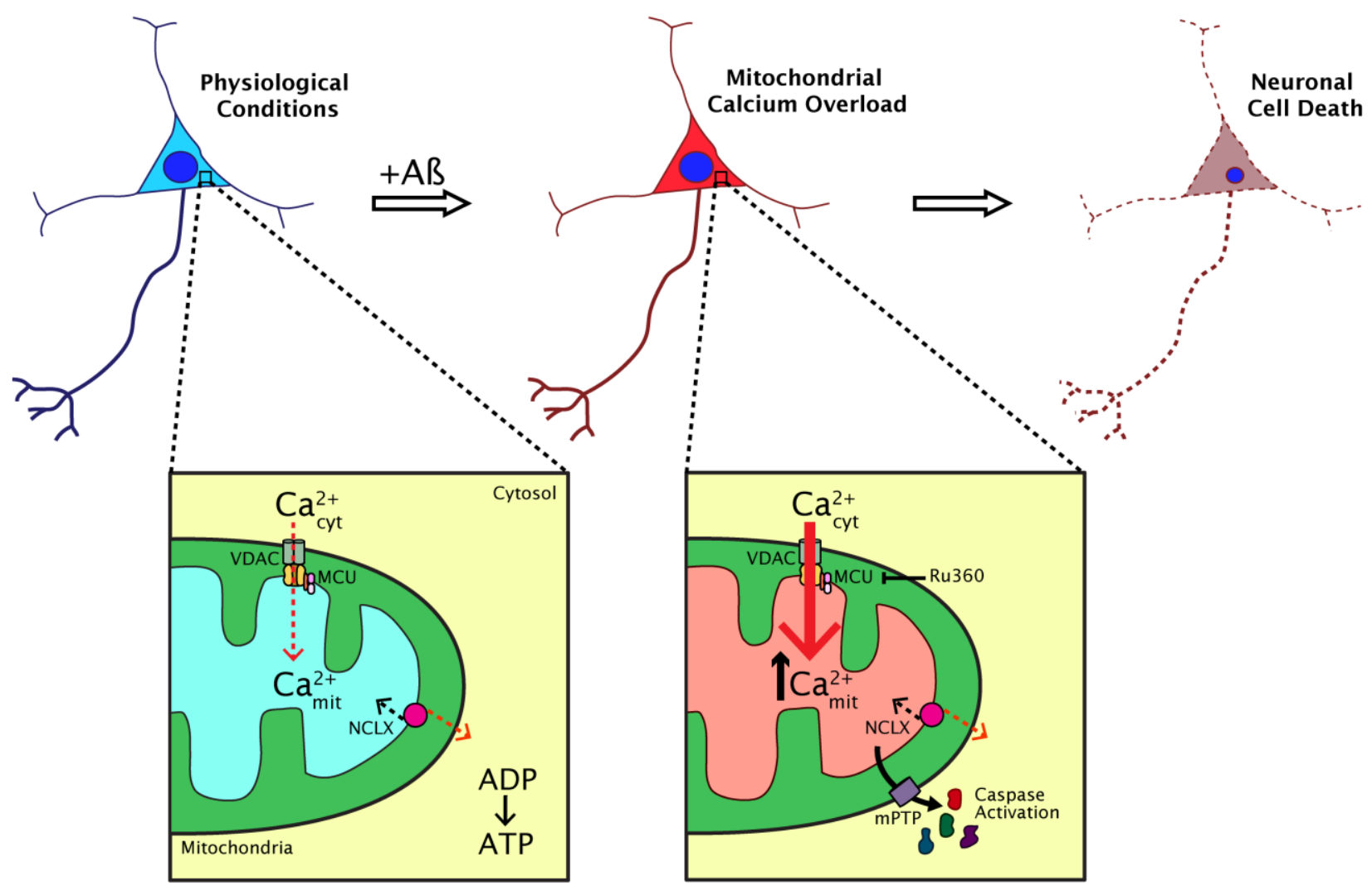

FIGURE 2: Major effects of $\mathbf{A} \boldsymbol{\beta}$ aggregates on mitochondrial $\mathrm{Ca}^{2+}$ homeostasis in neurons. In physiological conditions, mitochondria take up $\mathrm{Ca}^{2+}$ from the cytosol via the MCU to produce ATP among other functions. In AD, A $\beta$ aggregates (particularly soluble $A \beta$ ) increase mitochondrial $\mathrm{Ca}^{2+}$ via the $\mathrm{MCU}$ in vivo. This leads to mitochondrial $\mathrm{Ca}^{2+}$ overload and activates $\mathrm{mPTP}$ and caspases, which can trigger neuronal cell death via apoptosis. Blocking the MCU with Ru360 inhibits mitochondrial $\mathrm{Ca}^{2+}$ uptake, pointing to the $\mathrm{MCU}$ as a potential target for therapeutics aimed at preventing or reversing the progression of AD. 


\section{ACKNOWLEDGMENTS}

This work was supported by NIHR01AG0442603, S10 RR025645 and R56AG060974 (BJB), and by the Tosteson \& Fund for Medical Discovery and the BrightFocus Foundation A2019488F (MCR).

\section{CONFLICT OF INTEREST}

The authors declare no competing interests.

\section{COPYRIGHT}

(C) 2020 Calvo-Rodriguez and Bacskai. This is an openaccess article released under the terms of the Creative Commons Attribution (CC BY) license, which allows the unrestricted use, distribution, and reproduction in any medium, provided the original author and source are acknowledged.

Please cite this article as: Maria Calvo-Rodriguez and Brian J. Bacskai (2020). High mitochondrial calcium levels precede neuronal death in vivo in Alzheimer's disease. Cell Stress 4(7): 187-190. doi: 10.15698/cst2020.07.226 